\title{
Anthropometric and Biochemical Effects of Consuming Omega-3 Fatty Acids Enriched Eggs
}

\section{Adriana Beatriz Di iorio*, Freire Álvarez Brandon Javier and Vinueza Duque Rubén Esteban}

Department of Food Sciences, Zamorano University, Municipality of San Antonio de Oriente, Francisco Morazan, Honduras

*Corresponding Author: Adriana Beatriz Di iorio, Department of Food Sciences, Zamorano University, Municipality of San Antonio de Oriente, Francisco Morazan, Honduras.
Received: August 21, 2021

Published: September 18, 2021

(C) All rights are reserved by Adriana Beatriz Di iorio.

\begin{abstract}
Functional foods are products that control nontransmissible diseases. Egg is a low-cost food with high nutritional value and huge population intake. In the present study, the effect of Omega-3 fatty acids enriched eggs and non-enriched eggs on anthropometric measurements (weight, height, waist, hip, blood pressure, total fat percentage, visceral fat) and biochemical (lipid profile and glucose) were evaluated during 10 weeks, of 15 people with overweight or type I obesity. The participants were randomly assigned to receive a different treatment of enriched or non with Omega 3 eggs consumption with a dietary guideline or a specific eating plan. In the biochemical parameters, significant differences were observed for: total cholesterol $(p=0.037)$, HDL ( $p=0.001)$ in treatment T4 and triglycerides $(\mathrm{p}=0.033)$ in treatment T3. The adherence of each treatment was greater than $80 \%$. In conclusion, enriched Omega-3 fatty acid eggs intake showed significant changes in biochemical indicators as total cholesterol, triglycerides and increase of HDL concentration.
\end{abstract}

Keywords: Omega-3; Nontransmissible Diseases; Lipid Profile; HDL; Triglycerides

\section{Introduction}

Non-transmissible diseases (NTDs) are the leading cause of death worldwide, contributing to the demise of 41 million people each year or $71 \%$ of global deaths [1]. Among NTDs, weight-related disorders (both obese and overweight conditions) the leading causes of mortality.

Concerningly, the overweight and obesity rate in the Americas is $24.6 \%$, more than double the world average of $11.5 \%$ [2]. In Central America, one out of every five households includes at least one member who is overweight or obese. In Guatemala, Honduras and Costa Rica, $90 \%$ of individuals have a body fat percentage outside the normal range, with $70 \%$ of the population qualifying as overweight or obesity, $43 \%$ exhibiting high blood pressure, and at least $50 \%$ being physically inactive [3].
It is estimated that 60 to $70 \%$ of obese patients are dyslipidemic (high levels of plasma triglycerides, LDL and low levels of HDL) [4]. Atherogenic dyslipidemia (AD) is associated with inactive lifestyles, low fiber and high consumption of saturated and trans fatty acids. One way to counteract AD is with physical activity coupled with a diet low in carbohydrates and high in polyunsaturated fatty acids, such as Omega-3 fatty acids [5].

Omega-3 is an essential polyunsaturated fatty acid found in three dietary acids: alpha linolenic acid (ALA), eicosapentaenoic acid (EPA) and docosahexaenoic acid (DHA). ALA is found in vegetable oils such as flaxseed, soybeans, and canola while DHA and EPA are primarily found in fish such as tuna. Consumption of foods rich in ALA, EPA, or DHA increases circulating levels of Omega-3 in the body, thereby providing a litany of health benefits on car- 
diovascular disease (atrial fibrillation, atherosclerosis, thrombosis, inflammation, and sudden cardiac death), diabetes, cancer, depression and various mental illnesses, age-related cognitive decline, periodontal disease, and rheumatoid arthritis [6].

Between 2015 to 2020, the dietary guidelines for Americans recommended a consumption of 230 grams of fish varieties per week, with the express goal of consuming $250 \mathrm{mg}$ of EPA and DHA per day [7]. While consuming fish has numerous well-demonstrated nutritional benefits, per capita fish consumption among middle and lower-class families is low in Central American countries due to its high economic cost.

In contrast, eggs, particularly those derived from the common chicken (Gallus gallus domesticus) are a highly nutritious and affordable food source readily available throughout Central America. Eggs are rich in essential amino acids, which support the synthesis and maintenance of skeletal muscle mass [8]. Egg consumption in Central America has increased over the past years, as high production volumes and general favorability with consumers have led to its sale as a "commodity". While commonly available egg variety are low in Omega-3 fatty acids, supplementing poultry feed with flax seed can significantly increase the amount of ALA in chicken eggs. Theoretically, these 0mega-3 enriched eggs would accommodate the demand for this beneficial nutrient in addition to providing other benefits such as high protein value, all at an affordable cost to the consumer.

However, a lack of clinical trials at the regional level mean few affordable dietary alternatives exist to address the problems of obesity. Therefore, the following study aims to evaluate the biochemical and anthropometric changes following a diet supplemented with Omega-3 enriched eggs in people with overweight and obesity. Our findings suggest such a diet can improve their Body Mass Index, Blood Pressure, waist-hip index and their lipid profile (increase HDL, reduce cholesterol, triglycerides and LDL).

\section{Materials and Methods}

\section{Location and production of 0mega-3 enriched eggs}

The study was carried out at Zamorano Pan American Agricultural School, University of Agriculture, located in the Francisco Morazan Department, Honduras. Omega-3 enriched eggs were produced under the project "Effects of Zamofeed on the profile of fatty acids, cholesterol and pigmentation of the egg yolk". Adding flaxseed by $3 \%$ to the hens' diet increased the amount of $\alpha$-linolenic acid (ALA) resulting a product (egg) with an average mass of 60 grams, $325 \mathrm{mg}$ of Omega- 3 and a reduction of $27.8 \mathrm{mg}$ cholesterol relative to control eggs.

\section{Fatty acid profile of omega-3 enriched eggs}

To evaluate the profile of the eggs, the yolks were extracted and fatty acid (AOCS Ce 2-66 and AOCS Ce1j-07) and cholesterol (AOAC 994.10) content were determined via gas chromatography.

\section{Study description}

The clinical trial was previously approved by the Ethics Committee of Honduras belonging to the Autonomous University of Honduras. The research corresponds to an observational Phase I case-control clinical study. The sampling was non-probabilistic with desire to participate and the signing of an informed consent.

\section{Study population}

For the study, 16 workers of both sexes with ages between 28 and 47 years were selected via non-probabilistic random sampling. The selection criteria were: desire to participate voluntarily in the study, body mass index between $25-29.9 \mathrm{~kg}$ (overweight) or 30 $34.9 \mathrm{~kg}$ (Type I obesity), regular consumption of egg and not being allergic or intolerant to eggs. The excluded population were people with mental difficulties, body mass index $\leq 24.9$ or $\geq 35$, people intolerant or allergic to eggs.

\section{Organization of the study population}

Four participants were distributed in each treatment. The participants were randomly assigned to their treatment through the "Random" function of the Excel program. At the same time, each treatment was randomly assigned either a dietary guideline or a detailed, specific eating plan. The four treatments were:

T1. Five eggs without Omega-3 (Control) per person, per week, in conjunction with a specific eating plan.

T2. Three eggs without Omega-3 (Control) per person, per week, in conjunction with a dietary guideline.

T3. Five Omega-3 eggs (325 mg) per person, per week, in conjunction with a dietary guideline. 
T4. Three Omega-3 eggs (325 mg) per person, per week, in conjunction with a specific eating plan.

Evaluation of anthropometric measurements and blood pressure

Weight $(\mathrm{kg})$ and height $(\mathrm{m})$ were measured to determine the body mass index (IMC) $\left(\mathrm{kg} / \mathrm{m}^{2}\right)$. OMRON HBF-514C bioelectric impedance equipment was used to determine the weight. HBF-514C. A portable stadiometer 212 was used to determine height. To determine the waist-hip index (WHR = waist $\mathrm{cm} / \mathrm{hip} \mathrm{cm}$ ), waist and hip circumference measurements were collected using an inextensible SECA 201 tape measure. The values obtained, allowed the participants to be categorized, according to the ranges stipulated by the National Heart Lung Blood Institute [8].

To determine the body composition (percentage of fat and visceral fat), the OMRON bioelectric impedance equipment model HBF-514C was used, which consists of six low-voltage electrical conduction sensors. The values obtained were classified according to the "Organization World Health" [9] for body fat and Omron Healthcare [10] for visceral fat. Blood pressure was measured with an OMRON M3 digital blood pressure monitor, precision class 1 . The results provided by the cuff were categorized according to the "American Heart Association" [11].

Biochemical analysis (plasma lipid profile, glucose)

Total cholesterol, triglycerides, HDL and LDL were included in the lipid profile. Blood samples (35 uL) were taken from the participants via capillary and the DS Lipidocare portable was used to obtain the values of the mentioned lipid indicators. These values allowed the classification of the participants according to the ranges stipulated by the "National Institute of the Heart, Lung and Blood" [12]. The initial samples were collected as a reference value for comparison at the end of the study, after 10 weeks of the study. People fasted and did not smoke prior to data collection.

Blood sugar levels were determined using the ACCU-CHEK Rapid Test Kit. The sample was collected by capillary action using a test strip provided by the equipment manufacturer. To determine the category in which the participants were found, the ranges stipulated by the "National Institute of Diabetes and Digestive Kidney Diseases" [13].

\section{Adherence to treatment}

Participants' adherence to the assigned treatment was measured through the Morisky-Green test, which consists of a series of four questions with a dichotomous answer (yes/no). This questionnaire was applied in the middle of the study and at the end of the study.

\section{Statistical analysis}

The "Statistical Analysis System" (SAS®) version 9.4 program was used. The data were obtained through two measurements carried out in the same experimental unit, using a T-student test for paired samples and to evaluate the significance between treatments. A Duncan test was performed with a significance level of $P$ $\leq 0.05$.

\section{Results}

\section{Characterization of participants}

16 participants started the study. During the process one of them left the treatment, resulting in 15 total participants $(n=8$ men and $n=7$ women). Every participant was randomized and put in each of the four treatments. Then, people under control and treatments groups (Omega-3 enriched eggs), were observed in order to determinate the influence of eggs in the serum lipid profile.

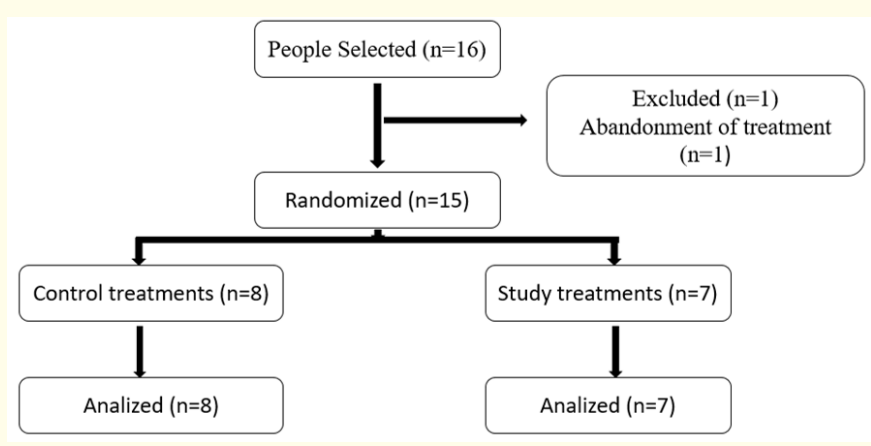

Figure 1: Flow chart of the participants in the study.

The anthropometric indicators evaluated were: age, height, weight, Body Mass Index (BMI), Waist-to-Hip-Ratio (WHR), systolic and diastolic blood pressure, glucose, total mass body fat, visceral fat, total cholesterol, triglycerides, HDL and LDL. 
Anthropometric and blood pressure measures

At the beginning of the study participants were overweight or had obesity type I (25 - 29.99 and $30-34.9 \mathrm{~kg} / \mathrm{m} 2$ ), with WHR between 0.92 and 1.00 ranges correlating to medium and high cardiometabolic risk, respectively. Treatment groups T1 and T3 displayed normal ranges of diastolic blood pressure $(<80 \mathrm{~mm} / \mathrm{Hg})$ however, treatments T2 and T4 had prehypertension ranges $(80-89 \mathrm{~mm} /$ $\mathrm{Hg}$ ). Values of total mass body fat and visceral fat were outside the normal ranges for all groups, with an average total mass body fat $(>33 \%)$ in women and $(>20 \%)$ in men and visceral fat percentage between (10-14\%) in the general population. The only exception was treatment group $\mathrm{T} 1$, which presented normal ranges of visceral fat $(\leq 9 \%)$.

End of study metrics were as follows. Participants who ate eggs enriched with Omega-3 (T3 and T4) exhibited reduced weight, BMI and WHR. Participants in group T2 reduced their systolic pressure to a normal range $(<120 \mathrm{~mm} / \mathrm{Hg})$ and participants in treatment $\mathrm{T} 1$ increased their levels of visceral fat to a high range (10-14\%), though this is comparable to the starting visceral fat for all other treatment groups. All these changes displayed statistical significance. Table 1 shows initial and final values of anthropometric measures and blood pressure for the four treatments.

\begin{tabular}{|c|c|c|c|}
\hline & \multicolumn{3}{|c|}{ Anthropometric measures } \\
\hline Variable & Initial & Final & P \\
\hline Weight $(\mathrm{kg})$ & & & \\
\hline T1 & $74.83 \pm 3.6$ & $75.20 \pm 6.2$ & 0.794 \\
\hline T2 & $79.43 \pm 12.0$ & $79.40 \pm 11.9$ & 0.965 \\
\hline T3 & $97.08 \pm 10.0$ & $95.33 \pm 10.1$ & 0.280 \\
\hline T4 & $99.50 \pm 24.4$ & $99.20 \pm 25.7$ & 0.777 \\
\hline Body Mass Index $(\mathrm{kg} / \mathrm{m} 2)$ & & & \\
\hline T1 & $28.50 \pm 2.9$ & $28.50 \pm 3.8$ & 1.000 \\
\hline T2 & $30.95 \pm 4.6$ & $31.00 \pm 4.4$ & 0.827 \\
\hline T3 & $33.43 \pm 3.5$ & $32.85 \pm 3.2$ & 0.352 \\
\hline T4 & $33.30 \pm 7.5$ & $33.20 \pm 7.8$ & 0.785 \\
\hline ICC & & & \\
\hline T1 & $0.92 \pm 0.0$ & $0.93 \pm 0.0$ & 0.391 \\
\hline T2 & $0.95 \pm 0.1$ & $0.94 \pm 0.0$ & 0.666 \\
\hline T3 & $0.95 \pm 0.1$ & $0.92 \pm 0.1$ & 0.194 \\
\hline T4 & $1.00 \pm 0.0$ & $0.96 \pm 0.0$ & 0.225 \\
\hline
\end{tabular}

\begin{tabular}{|c|c|c|c|}
\hline \multicolumn{4}{|c|}{$\begin{array}{c}\text { Systolic blood pressure } \\
(\mathrm{mm} / \mathrm{Hg})\end{array}$} \\
\hline $\mathrm{T} 1$ & $111 \pm 20.3$ & $109 \pm 19.1$ & 0.576 \\
\hline $\mathrm{T} 2$ & $125 \pm 15.9$ & $116 \pm 9.1$ & 0.117 \\
\hline T3 & $111 \pm 4.0$ & $105 \pm 9.9$ & 0.438 \\
\hline $\mathrm{T} 4$ & $117 \pm 10.0$ & $119 \pm 13.0$ & 0.713 \\
\hline \multicolumn{4}{|c|}{$\begin{array}{l}\text { Diastolic blood pressure } \\
(\mathrm{mm} / \mathrm{Hg})\end{array}$} \\
\hline $\mathrm{T} 1$ & $76 \pm 14.0$ & $82 \pm 10.2$ & 0.512 \\
\hline $\mathrm{T} 2$ & $80 \pm 19.6$ & $72 \pm 7.6$ & 0.432 \\
\hline $\mathrm{T} 3$ & $75 \pm 3.9$ & $74 \pm 6.7$ & 0.876 \\
\hline $\mathrm{T} 4$ & $82 \pm 6.0$ & $92 \pm 11.5$ & 0.453 \\
\hline \multicolumn{4}{|c|}{$\begin{array}{l}\text { Body corporal total fat } \\
(\%)\end{array}$} \\
\hline $\mathrm{T} 1$ & $36.10 \pm 9.2$ & $33.93 \pm 8.7$ & 0.471 \\
\hline $\mathrm{T} 2$ & $38.30 \pm 11.9$ & $38.60 \pm 10.9$ & 0.617 \\
\hline $\mathrm{T} 3$ & $40.85 \pm 11.6$ & $41.08 \pm 11.1$ & 0.685 \\
\hline $\mathrm{T} 4$ & $37.83 \pm 12.3$ & $37.77 \pm 13.3$ & 0.930 \\
\hline \multicolumn{4}{|c|}{ Visceral fat (\%) } \\
\hline $\mathrm{T} 1$ & $9.00 \pm 2.2$ & $10.25 \pm 1.0$ & 0.391 \\
\hline $\mathrm{T} 2$ & $10.75 \pm 3.3$ & $11.00 \pm 3.6$ & 0.391 \\
\hline T3 & $11.25 \pm 2.8$ & $10.75 \pm 2.9$ & 0.181 \\
\hline $\mathrm{T} 4$ & $12.33 \pm 3.5$ & $12.00 \pm 4.0$ & 0.422 \\
\hline
\end{tabular}

Table 1: Initial and final values of anthropometric measures and blood pressure for all treatment groups.

$\pm \mathrm{DE}$ represents media values, $(\mathrm{P}<0.05)$ shows significant changes between initial and final measures.

\section{Biochemical measures}

At the beginning of the study, treatment T4 displayed normal ranges of blood glucose $(<120 \mathrm{mg} / \mathrm{dL})$ while the other treatment groups presented ranges of prediabetes (100-125 md/dL). Treatment group T4 exhibited high total cholesterol (200-239 mg/dL), while the other treatment groups were below the normal threshold $(<200 \mathrm{mg} / \mathrm{dL})$.

Groups T1-T3 displayed higher concentrations of triglycerides (200-499 mg/dL), while treatment T4 was in a normal range $(<150 \mathrm{mg} / \mathrm{dL})$. Concentrations of HDL amongst the four treatment groups were low ( $<40 \mathrm{mg} / \mathrm{dL}$ ), as were the LDL values $(<100 \mathrm{mg}$ / $\mathrm{dL}$ ), with only treatment $\mathrm{T} 1$ presenting an optimal concentration of LDL (100-129 mg/dL). 
Blood glucose levels were unchanged following the conclusion of the study. Similarly, lipid profiles and total cholesterol values did not present significant changes, although nearly all treatments trended towards a decrease in these metrics, with the largest reductions occurring in treatments T3 and T4. Only treatment T1 demonstrated an increase in total cholesterol, changing their category from desirable to high (200-239 mg/dL).

Triglycerides showed a significant reduction in treatments T3 (268.50 to $190.35 \mathrm{mg} / \mathrm{dL}$ ) changing its category from high, to high-limit and T4 (379.33 to $274.45 \mathrm{mg} / \mathrm{dL}$ ), which maintained its category. In order to decrease cardiovascular risks HDL values must be more than $50 \mathrm{mg} / \mathrm{dl}$. Patients in treatment groups T3 and T4 nearly achieved this protective value. Although treatment T1 was close to $50 \mathrm{mg} / \mathrm{dl}$, treatment $\mathrm{T} 4$ stood out due to its greater increase relative to its initial value. After study completion, every treatment belonged to the normal HDL category $(40-60 \mathrm{mg} / \mathrm{dL})$.

LDL was modestly reduced in participants who ingested Omega-3 eggs relative to the control treatments. However, none of the treatments presented significant differences.

\begin{tabular}{|c|c|c|c|}
\hline & \multicolumn{3}{|c|}{ Biochemical measures } \\
\hline Variable & Initial & Final & $\mathbf{P}$ \\
\hline \multicolumn{4}{|c|}{ Glucose (mg/dL) } \\
\hline $\mathrm{T} 1$ & $105 \pm 6.4$ & $107 \pm 6.7$ & 0.736 \\
\hline $\mathrm{T} 2$ & $105 \pm 17.3$ & $108 \pm 14.2$ & 0.775 \\
\hline T3 & $103 \pm 4.1$ & $111 \pm 8.6$ & 0.197 \\
\hline $\mathrm{T} 4$ & $98 \pm 5.0$ & $91 \pm 12.9$ & 0.524 \\
\hline \multicolumn{4}{|c|}{ Total cholesterol (mg/dL) } \\
\hline T1 & $182.25 \pm 47.3$ & $202.14 \pm 60.2$ & 0.085 \\
\hline $\mathrm{T} 2$ & $176.00 \pm 37.6$ & $172.13 \pm 28.0$ & 0.691 \\
\hline T3 & $159.00 \pm 34.7$ & $146.20 \pm 19.7$ & 0.220 \\
\hline $\mathrm{T} 4$ & $205.67 \pm 38.6$ & $198.05 \pm 40.5$ & 0.037 \\
\hline \multicolumn{4}{|c|}{ Triglycerides (mg/dL) } \\
\hline T1 & $177.25 \pm 43.8$ & $168.95 \pm 39.2$ & 0.482 \\
\hline $\mathrm{T} 2$ & $307.75 \pm 78.6$ & $250.99 \pm 106.7$ & 0.096 \\
\hline T3 & $268.50 \pm 185.0$ & $190.35 \pm 162.9$ & 0.033 \\
\hline $\mathrm{T} 4$ & $98.20 \pm 5.7$ & $93.86 \pm 1.1$ & 0.383 \\
\hline \multicolumn{4}{|l|}{ HDL (mg/dL) } \\
\hline $\mathrm{T} 1$ & $38.00 \pm 8.6$ & $49.30 \pm 2.5$ & 0.034 \\
\hline $\mathrm{T} 2$ & $30.50 \pm 7.5$ & $48.88 \pm 3.7$ & 0.021 \\
\hline T3 & $37.50 \pm 8.3$ & $49.51 \pm 2.8$ & 0.037 \\
\hline $\mathrm{T} 4$ & $31.33 \pm 3.2$ & $49.30 \pm 4.5$ & 0.001 \\
\hline LDL (mg/dL) & & & \\
\hline
\end{tabular}

\begin{tabular}{|c|c|c|c|}
\hline T1 & $108.60 \pm 51.6$ & $119.05 \pm 63.3$ & 0.192 \\
\hline T2 & $83.75 \pm 20.9$ & $73.05 \pm 26.4$ & 0.392 \\
\hline T3 & $67.80 \pm 13.1$ & $58.62 \pm 14.4$ & 0.368 \\
\hline T4 & $98.20 \pm 5.7$ & $93.86 \pm 1.1$ & 0.383 \\
\hline
\end{tabular}

Table 2: Initial and final values of biochemical measures of the four treatments.

\pm DE represents media values, $(\mathrm{P}<0.05)$ shows significant changes between initial and final measures.
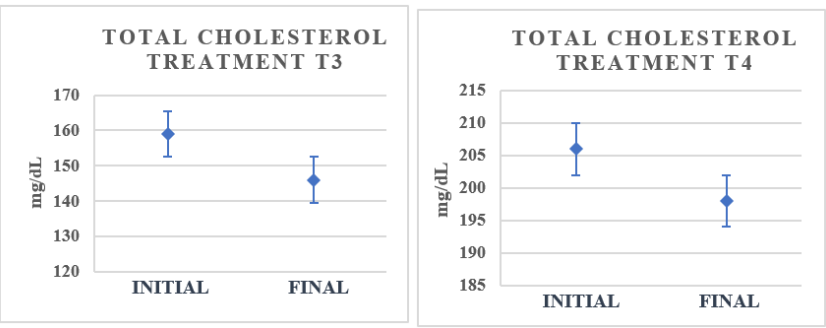

Figure 2: Initial and final values of total cholesterol for treatments T3 and T4.

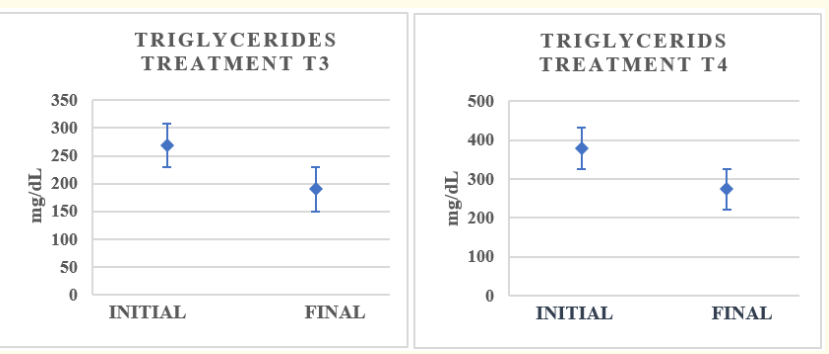

Figure 3: Initial and final values of triglycerides for treatments T3 and T4.

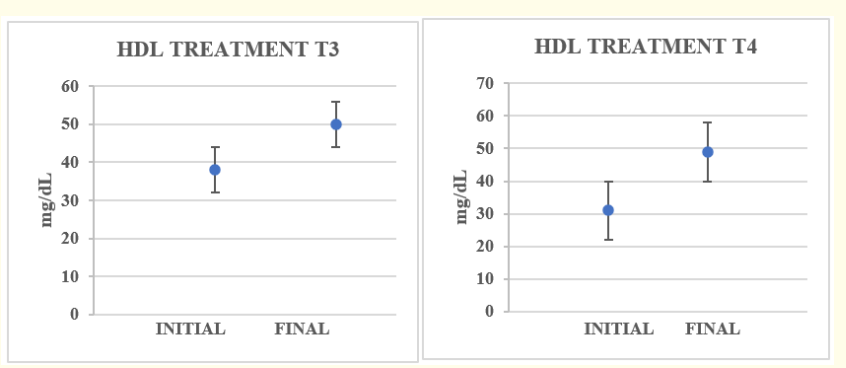

Figure 4: Initial and final values of HDL for treatments T3 and T4. 


\section{Adherence to treatment}

Morisky-Green results are presented on figure 2 as percentages. Every treatment had a greater adherence than $80 \%$, with treatment T3 demonstrating the highest percentage adherence.

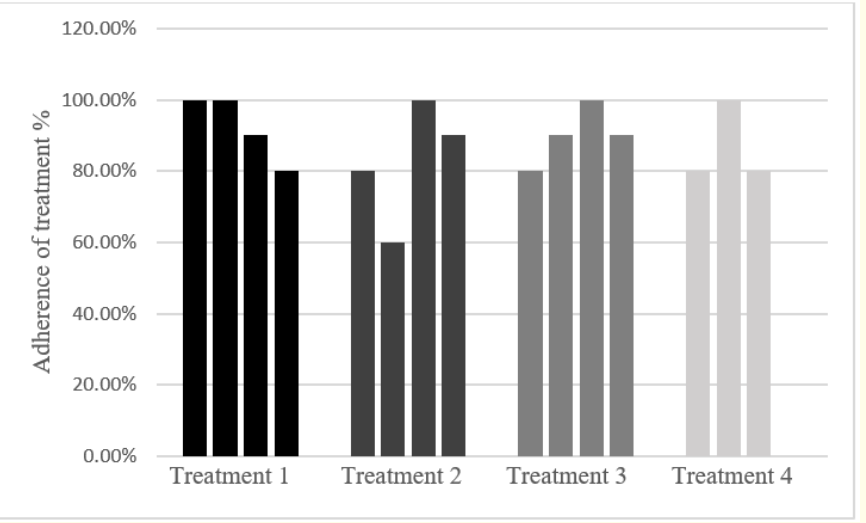

Figure 5: Treatment adherence per treatment.

\section{Discussion}

Our results suggest that the addition of flaxseed and phytosterols in poultry diets may provide health benefits to obese and overweight patients, likely due to the improved nutritional value of the eggs. The modification of poultry diet increase the polyunsaturated acids Omega-3 content in the egg yolk [14]. The polyunsaturated acids are macromolecules, which have a crucial role in the prevention and protection of people at risk for cardiovascular illnesses [15]. Lemahieu., et al. [16] determined that a flaxseed diet in poultry significantly increases linolenic, EPA and DHA in egg yolks.

This study showed that normal eggs and Omega-3 enriched eggs did not significant change the anthropometric measures of the participants. This statement agrees with a study of Ahmad., et al. [17], which concluded that a diet including Omega-3 enriched eggs did not significantly affect patient BMI.

Patients ingesting Omega-3 enriched eggs also did not display significant differences in blood pressure. However, treatment T3, which included enriched eggs with Omega-3 diet, demonstrated the greatest reduction in systolic pressure. Omega-3 decreases oxidative stress by inhibiting the formation of reactive oxidative species (ROS) and isoprostanes. This reduction increases the bio- availability of nitric oxide, which in turn leads to vasodilatation and a reduction in blood pressure [18]. Excess ROS can alter cellular function, irreversibly damaging lipids, nucleic acids and cellular proteins. This oxidative damage can lead to numerous pathologies including hypertension, insulin resistance, neurodegenerative illnesses and so on [19]. Isoprostanes, including the especially potent F2, are strong vasoconstrictors [20].

Other potential benefits of consuming a diet rich in Omega-3 are derived in the fact that DHA can directly stimulate vascular smooth muscle through increased activity of $\mathrm{K}+$ channels, which are dependent on $\mathrm{Ca} 2+$ ions. This activation leads to dilatation of blood vessels, which reduce blood pressure and improve vascular health [21]. Calcium and potassium channels are responsible for the contraction and relaxation, or vasodilation of blood vessels, respectively [22].

The lack of significant changes in blood glucose levels following dietary supplementation with Omega-3 enriched eggs is not surprising, given the fact that Omega-3 does not affect plasmatic glucose levels, nor HbA1c [23]. Despite this, treatment T4 reduced glucose levels, thereby maintaining a normal range throughout the study. Of note, while patients in group T4 suffered from type I obesity, which is frequently correlated with prediabetes [24], all blood glucose levels were in the normal range.

Increase of adiposity and free fatty acids of obese patients leads to a homeostatic response which drives insulin resistance and hyperinsulinemia [25]. Insulin resistance is defined by ineffective insulin secretion, leading to nonresponsive insulin receptor cells. Insulin hypersecretion in the early stages of disease maintain relatively normal glycemic indices, leading to underreported cases of prediabetes [26]. These data suggest that while patients in treatment T4 may have had (pre-)diabetes, no metabolic anomalies could be detected using the tools and techniques available.

Following study, omega-3 did corporeal fat values in either men or women through completion of the study. Instead, each treatment group maintained high levels of corporeal fat, which fits with previous studies exploring this [27]. While we observed a reduction of visceral fat in treatments T3 and T4, these findings lack statistical significance. A previous study by Mello., et al. [28] confirms these findings: incorporation of Omega-3 in a high fat diet of obese mice after 10 weeks, leads to, observed beneficial effects in the reduction of visceral fat. 
Some studies showed that Omega-3 fatty acids had the potential to reduce accumulated fat in the body, especially visceral fat [29]. Their proposed mechanism of action is that Omega-3 reduces oxidative damage and relieves changes in the antioxidant defense system and Krebs cycle, also Omega-3 to mitigate changes which could lead to a high fat diet [30].

The treatment groups receiving Omega-3 enriched eggs (T3 and T4), both displayed reductions in their cholesterol total values; however, only treatment T4 showed a statistically significant reduction. These findings align with the results from Shakoor., et al. [31], who demonstrated that the intake of two eggs enriched with Omega-3 for 5 weeks had the capability to reduce cholesterol by $12.5 \%$. While the mechanism of action for the effect is still unclear, one study suggests that EPA confers a hypocholesterolemic effect by increasing bile flow and reverse transportation of cholesterol [32].

The National Lipid Association recommends a diet rich in Omega-3 fatty acids for patients with hypotriglyceridemic, as studies have shown this may decrease triglycerides levels. Our findings confirm the results of a previous study, where consuming Omega-3 enriched eggs significantly decreased triglycerides levels. Omega-3 fatty acids have low VLDL (very low-density lipoprotein) incorporation rates, which may increase the elimination rates of triglycerides in VLDL particles. Triglycerides are amphipathic molecules which assembly in blood lipoproteins (VLDL and chylomicrons) for transportation through the blood plasma to satisfying energetic needs.

Polyunsaturated fatty acids (PUFA) are high quality fats which benefit overall health. One study has shown that enriching eggs with PUFAs help to decrease LDL cholesterol. High density lipoproteins (HDL) synthetized in the gut and liver remove cholesterol of peripheral tissues, thereby conferring protection against atherogenesis. General consumption of eggs (Omega-3 enriched or not) is known to increase HDL levels.

In contrast, multiple studies have found that frequent consumption of eggs does not significantly alter levels of LDL cholesterol. However, our results from treatments $\mathrm{T} 3$ and $\mathrm{T} 4$ demonstrate a reduction in LDL, albeit without statistical significance. This reduction may be attributed to enriched Omega-3 consumption. A study by Valenzuela., et al. highlighted the beneficial effects on cardiovascular health conferred by polyunsaturated fatty acids Omega-3, which they argued was due to reduced LDL cholesterol.
To confirm the adherence by study participants, we performed clinical or therapeutic interviews, wherein an interviewer evaluates the effectivity and security of the prescribed treatments. To consider a treatment adherent, the medicine or treatment intake must be greater than $80 \%$, while less than $80 \%$ is consider nonadherent. Every treatment group displayed greater than $80 \%$ adherence, allowing all treatment groups to be included as part of the study.

\section{Conclusion}

Enriched Omega-3 eggs intake showed significant changes in biochemical indicators. For total cholesterol profile $(p=0.037)$, triglycerides $(\mathrm{p}=0.033)$ and increase of HDL concentration $(\mathrm{p}=$ 0.001).

\section{Conflict of Interest}

The authors express that there are no conflicts of interest when writing the manuscript.

\section{Acknowledgement}

Thank you to ITHC: the Institute for Technology in Health Care that was good to fund the biochemical analysis.

\section{Bibliography}

1. World Health Organization. Non-communicable diseases (2018).

2. Medici A., et al. "The economic dimensions of Non-transmissible diseases in Latin America and the Caribbean". Washington D.C: OPS. (2017).

3. INCAP. Annual work report. (2016).

4. Kenneth R and Feingold MD. Obesity and Dyslipidemia (2020).

5. Ponte C., et al. "Atherogenic dyslipidemia in Latin America: prevalence, causes and treatment". 15 (2017): 1690-3110.

6. Zhang Z., et al. "Dietary Intakes of EPA and DHA Omega-3 Fatty Acids among US Childbearing-Age and Pregnant Women: An Analysis of NHANES 2001-2014". In Nutrients. 10.4 (2018).

7. Dussaillant C., et al. "Egg intake and cardiovascular disease: a scientific literature review". Nutrición Hospitalaria 34.3 (2017): 710-718.

8. National Heart, Lung and Blood Institute. Managing Overweight and Obesity in Adults: Systematic Evidence Review from the Obesity Expert Panel (2013). 
9. American Heart Association. Blood (2016).

10. National Institute of Diabetes and Digestive Kidney Diseases. Know your blood sugar numbers: Use them to manage your diabetes (2016).

11. Laudadio V., et al. "Enhancement of Nutraceutical Value of Table Eggs Through Poultry Feeding Strategies". International Journal of Pharmacology 11 (2015): 201-212.

12. Lavie C., et al. "Obesity and cardiovascular disease: risk factor, paradox, and impact of weight loss". Journal of the American College of Cardiology 53.21 (2009): 1925-1932.

13. Lemahieu C., et al. "Impact of different omega-3 polyunsaturated fatty acid (n-3 PUFA) sources (flaxseed, Isochrysis galbana, fish oil and DHA Gold) on n-3 LC-PUFA enrichment (efficiency) in the egg yolk". Journal of Functional Foods 19 (2015): 821-827.

14. Ahmad M., et al. "Cardiovascular Disease Risk and its Association with Body Mass Index in Malaysians Based on the World Health Organization/International Society of Hypertension Risk Prediction Chart". Health Science Journal 12.1 (2018).

15. Stupin A., et al. "Omega-3 polyunsaturated fatty acids-enriched hen eggs consumption enhances microvascular reactivity in young healthy individuals". Applied Physiology, Nutrition, and Metabolism 43.10 (2018): 988-995.

16. Subramanian S and Chait A. "Hypertriglyceridemia secondary to obesity and diabetes". Biochimica et Biophysica Acta 1821.5 (2012): 819-825.

17. Ros M and Medina-Gómez G. "Obesity, adipogenesis and insulin resistance”. Endocrinology Nutrition 58.7 (2011): 360-369.

18. Haas A., et al. "Omega-3 polyunsaturated fatty acids have beneficial effects on visceral fat in diet-induced obesity model". Biochemistry and Cell Biology 97.6 (2019).

19. Mozaffarian D and Wu JHY. “ (n-3) fatty acids and cardiovascular health: are effects of EPA and DHA shared or complementary?" The Journal of Nutrition 142.3 (2012): 614S-625S.

20. Shakoor H., et al. "Development of Omega-3 rich eggs through dietary flaxseed and bio-evaluation in metabolic syndrome". Food Science and Nutrition 8.6 (2020): 2619-2626.

21. Stone N., et al. "ACC/AHA guideline on the treatment of blood cholesterol to reduce atherosclerotic cardiovascular risk in adults: a report of the American College of Cardiology/American Heart Association Task Force on Practice Guidelines". Circulation 129 (2014): S1-45.
22. Backes J., et al. "The clinical relevance of Omega-3 fatty acids in the management of hypertriglyceridemia". Lipids Health Disease 15.1 (2016): 118.

23. Handelsman Y and Shapiro M. "Triglycerides, atherosclerosis, and cardiovascular outcome studies: focus on Omega-3 fatty acids". Endocrine Practice 23.1 (2017): 100-112.

24. Backes J., et al. "The clinical relevance of Omega-3 fatty acids in the management of hypertriglyceridemia". Lipids Health Disease 15.1 (2016): 118.

25. Fraeye I., et al. "Dietary enrichment of eggs with Omega-3 fatty acids: A review”. Food Research International 48.2 (2012): 961-969.

26. Puga G., et al. "High density lipoproteins and cardiovascular risk". REB 38.4 (2019): 93-99.

27. Khan S., et al. "Comparative study of fatty-acid composition of table eggs from the Jeddah food market and effect of value addition in Omega-3 bio-fortified eggs". Saudi Journal of Biological Sciences 24.4 (2017): 929-935.

28. DiMarco D and Fernandez M. "Differences in response to eggderived dietary cholesterol result in distinct lipoprotein profiles while plasma concentrations of carotenoids and choline are not affected in a young healthy population". Journal of Agriculture and Food Research 1 (2019).

29. Klangjareonchai T., et al. "The effect of ingestion of egg and low-density lipoprotein (LDL) oxidation on serum lipid profiles in hypercholesterolemic women". Songklanakarin Journal of Science and Technology 34.2 (2012): 173-178.

30. Valenzuela B., et al. "Omega-3 fatty acids (EPA and DHA) and their application in various clinical situations". Revista Chilena de Nutricion 38.3 (2011): 356-367.

31. Rodríguez M., et al. "Review of the test used for measuring therapeutic compliance in clinical practice". Aten Primaria 40.8 (2008): 413-418.

32. Lam W and Fresco P. "Medication Adherence Measures: An Overview". BioMed Research International (2015).

Volume 4 Issue 10 October 2021 (C) All rights are reserved by Adriana Beatriz Di iorio., et al. 